УДК: 538.911

DOI: $10.18384-2310-7251-2017-3-76-83$

\title{
УПРАВЛЯЕМЫЕ ДИФРАКЦИОННЫЕ ЭЛЕМЕНТЫ НА ОСНОВЕ ЖИДКОКРИСТАЛЛИЧЕСКИХ КОМПОЗИТОВ
}

\author{
Соломатин А.С. ${ }^{1}$, Мащенко В.И. , Беляев В.В. , Маргарян А.Л. ${ }^{2}$, Акопян Н.Г. \\ 1 Московский государственный областной университет, \\ учебно-научная лаборатория теоретической и прикладной нанотехнологии \\ 105005, г. Москва, ул. Радио, д. 10А, Российская Федерация \\ 2 Ереванский государственный университет, \\ центр полупроводниковых приборов и нанотехнологий \\ 0025, г. Ереван, ул. Алекса Манукяна, д. 1, Республика Армения
}

Аннотация. Авторами предложены новые управляемые ЖК-композитные дифракционные оптические элементы на основе нового явления формования в матрице боросилоксана микронитей ЖК с длинной до нескольких сантиметров. Материалы на базе исследованных ЖК-композитов могут быть использованы в дисплейной технике и оптоэлектронике, например, в качестве микрорезонаторов и электронно-оптических датчиков.

Ключевые слова: жидкокристаллический композит, нематический жидкий кристалл, боросилоксан, микроструктура, дифракция.

\section{CONTROLLABLE LC-COMPOSITE DIFFRACTIVE OPTICAL ELEMENTS}

\author{
A. Solomatin ${ }^{1}$, V. Mashchenko', V. Belyaev', A. Margaryan², N. Hakobyan² \\ 1 Moscow Region State University, \\ Education \& Science Lab for Theoretical and Applied Nanotechnology \\ ul Radio 10A, 105005 Moscow, Russian Federation \\ 2 Yerevan State University, \\ Center of Semiconductor Devices and Nanotechnologies \\ 1, Alex Manoogian st., Yerevan, 0025, Republic of Armenia
}

Abstract. We propose new controllable LC-composite diffractive optical elements based on a new phenomenon of forming LC microfilaments with a length of up to several centimeters in a borosiloxane matrix. Materials based on the investigated LC composites can be used in display technology and optoelectronics, for example, as microresonators and electron-optical sensors.

Key words: liquid crystal composite, neumatic liquid crystal, borosiloxane, microstructure, diffraction.

\section{Введение}

Широкое применение в дисплейной технике и других устройствах нашли ЖК и композиты на их основе (ЖК-композиты) [2; 9]. Получению и исследованию

(c) Соломатин А.С., Мащенко В.И., Беляев В.В., Маргарян А.Л., Акопян Н.Г., 2017. 
ЖК-композитов, где ЖК равномерно диспергированы в инертной среде, например, в полимерной матрице, посвящено большое количество работ [2; 11]. Формированию в таких композитах микроструктуры, которая бы обусловила получение требуемых макрохарактеристик материала или устройства придаётся большое значение [10].

Системы, где отдельная микронить или микроучасток поверхности ЖК используется в качестве функционального элемента микроустройства, представляют научный и практический интерес наряду с макрохарактеристиками материалов ввиду широкого развития миниатюризации, микро- и нанотехнологий.

Силиконовые масла и полимеры часто используются в широком интервале температур в качестве инертной среды для размещения капли ЖК ввиду их высокой прозрачности (вследствие аморфности структуры), инертности, постоянства параметров и технологичности. Силиконы обладают хорошей теплопередачей, являются отличными электрическими изоляторами, не являются легковоспламеняющимися, обладают эластичностью, долговечностью, био- и химической инертностью, экологичностью. Они обладают показателем преломления около 1,4 , что меньше показателя преломления большинства традиционных ЖК (обычно более 1,5).

Получают боросилоксан (БС) реакцией силоксанов с борангидридом, борной кислотой и др. соединениями бора. Боросилоксан, сохраняя все достоинства силиконовых материалов, интересен в качестве матрицы ЖК-композита, т. к. он обладает каучукоподобной способностью к растяжению при резком воздействии и маслообразной текучестью при статической нагрузке, т. е. уникальными механическими свойствами дилатантной жидкости.

Целью данной работы является разработка новых управляемых ЖКкомпозитных дифракционных оптических элементов на основе боросилоксана.

\section{Экспериментальная часть}

Боросилоксан получен по методике, адаптированной для данной задачи согласно патенту РФ [3].

В качестве ЖК была использована нематическая смесь ЖК-1282 ( $\mathrm{T}_{\mathrm{CN}}=253,1 \mathrm{~K}$; $\left.\mathrm{T}_{\mathrm{NI}}=335,1 \mathrm{~K}\right)$, которая состоит из алкоксицианбифенилов (80\% массовой доли), эфира Демуса (16\%) и эфира Грея (4\%) производства ФГУП «НИОПИК» (РФ).

\section{Микроструктура и оптические свойства ЖК-композитов}

Боросилоксан представляет собой неньютоновскую (дилатантную) жидкость, которая при отсутствии внешнего напряжения ведёт себя подобно вязкому силиконовому маслу, растекаясь по поверхности подложки, а при растяжении ведёт себя подобно жевательной резинке.

ЖК-композит является молочно-белым, в то время как исходный боросилоксан является прозрачным материалом, напоминающим силиконовый герметик. Важно отметить, что у БС, наполненного 40 масс. \% ЖК, способность к вытяжке сохраняется. Окраска ЖК-композита связана, скорее всего, с обусловленным разницей в показателях преломления рассеянием на границе раздела фаз БС - 
ЖК. По-видимому, данное явление связано с микроструктурой композита, где, не нарушая целостность боросилоксановой матрицы, изолированно друг от друга распределены капли ЖК.

На данный способ получения ЖК-композитов подана заявка на патент РФ [1; 6]. В настоящий момент решение о выдаче патента РФ уже принято Федеральным институтом промышленной собственности.

Исходный БС, как показали микроскопические исследования в поляризованном свете, не обладает двулучепреломлением. Таким образом, структура данного композита, где капли ЖК распределены в матрице боросилоксана, является двухфазной.

Структура композитов, по-видимому, такова, что силиконовый материал, обволакивая и смачивая ЖК во внутреннем объёме, постоянно присутствует только на поверхности. Композит имеет показатель преломления на уровне концентрации ЖК в материале 2,13 масс. \% при содержании ЖК 41,2 масс. \%. По-видимому, при комнатной температуре в боросилоксане 2,13 масс. \% - это то количество ЖК, которое растворяется, что в целом хорошо согласуется с литературными данными. Следует отметить, что, по данным научного источника [7], растворимость в силиконах ЖК близкого химического состава обычно составляет около 4 масс. \%.

\section{Механизм образования и разрушения микрофиламентов ЖК}

Была предложена схема для описания процесса образования цилиндров (рис. 1) и капель ЖК. К формированию капель ЖК в объёме БС приводит изначальное механическое смешивание компонентов. Далее капли сильно вытягиваются за счёт механического воздействия (одноосного растяжения), прикладываемого к композиту, становясь длинными цилиндрами с заострёнными конусами на концах (нитевидными объектами).

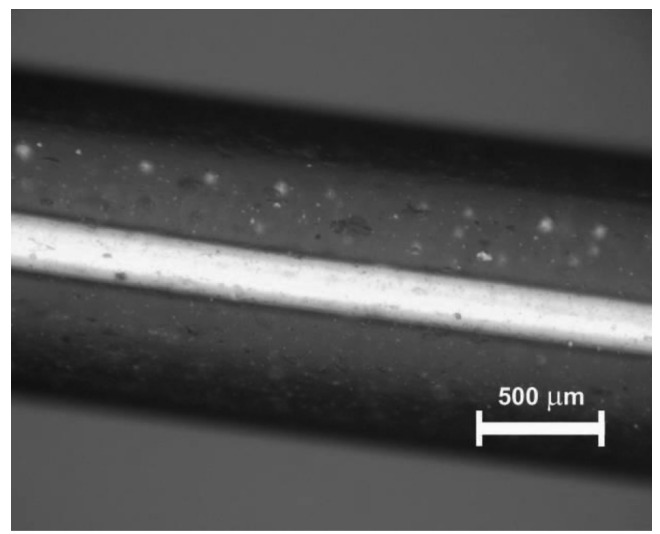

Puc. 1. Микрофотография в скрещённых поляризаторах вытянутого образца БС, наполненного ЖК-1282. Видны выстроенные вдоль одной линии микрокапли уже разделившегося более тонкого микрофиламента ЖК в БС, а также яркий микрофиламент 
Далее, когда механическое воздействие прекращается, БС перестаёт вести себя подобно эластомеру и превращается в вязкую жидкость. Начинает проявляться известный эффект деления растянутой капли внутри другой несмешиваемой с ней жидкости [8]. Однако длину таких нитей до нескольких сантиметров обычно не удавалось получить. Длина вытянутой нити ЖК практически равна длине всего вытянутого объекта. В нашем случае при толщине нити около 2-10 мкм длина нити доходит до 10 и более сантиметров.

\section{Неоднородные композитные структуры, формирующие твист-ячейку}

Можно предложить способ изготовления твист-структуры на основании полученных экспериментальных результатов. Содержащую тонкий ЖК цилиндр композитную нить следует изготавливать короткой (длиной меньше 1 мм). Затем следует повернуть на $\pi / 2$ один её конец. В результате при прохождении луча света вдоль ЖК цилиндра будет происходить поворот плоскости поляризации, как в $90^{\circ}$ твист-ячейке. При меньшем угле закрутки поворот плоскости поляризации будет, соответственно, меньше.

Предлагаемая в данной работе ЖК структура изображена на рис. 2. Она представляет собой подобную описанной в [5] матрицу из цилиндрических пор с нитями вышеописанного (рис. 1) композита в них. Это установленный перед дифракционной решёткой упорядоченный блок ЖК твист-ячеек.

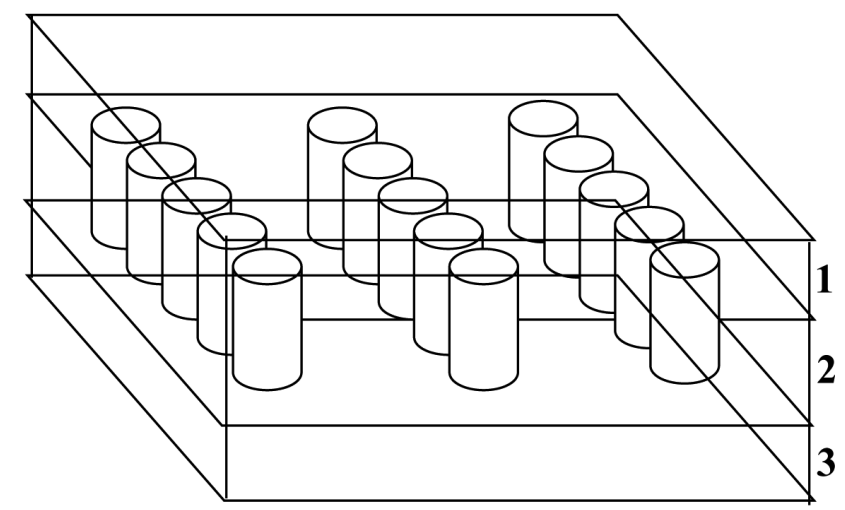

Puc. 2. Блок ЖК твист-ячеек. Обозначения: 1 - прозрачный слой сверху

(перед матрицей твист-ячеек); 2 - непрозрачный слой, содержащий блок твист-ячеек (цилиндрические поры, заполненные ЖК); 3 - прозрачный слой, отделяющий блок от дифракционной решётки. Решётка не показана на рисунке.

Свет падает сверху вниз

\section{Композитные матричные структуры с контролем поляризации падающего на дифракционную решётку луча света}

Дифракционные свойства оптически анизотропных полимерных структур периодическим микрорельефом были смоделированы в [4] для различных типов периодических микроструктур. 
В [6] были рассчитаны характеристики пропускания поляризованного света цилиндрическими анизотропными ЖК структурами в оптически изотропном окружении.

Как следует из $[4 ; 6]$, можно изготовить дифракционную решётку из параллельно расположенных нитей вышеописанного ЖК-композита на основе борсилоксана таким образом, что свет будет проходить только через анизотропные цилиндрические структуры.

Управление поляризацией падающего на дифракционную решётку луча света может быть осуществлено с помощью матричной структуры, предложенной выше. На рис. 3 показан блок из четырёх элементов с различной поляризацией луча на выходе, благодаря различному углу твист-закрутки. На такие блоки разбивается матричная структура. Каждый блок задаёт поляризацию проходящего через него луча света, регулируя пропускание составляющих блок твист-ячеек электрическим полем. Затем луч света с заданной поляризацией поступает на дифракционную решётку.

Управление интенсивностью света, с той или иной поляризацией падающего на дифракционную решетку, осуществляется следующим образом. Каждая твист-ячейка имеет на выходе анализатор, плоскость пропускания которого соответствует плоскости поляризации выходящего из данной ячейки света. При приложении к твист-ячейке управляющего поля интенсивность пропускания будет снижаться. Для обеспечения полного погашения света ячейкой, необходимы углы закрутки твиста больше или равные $\pi / 2$.

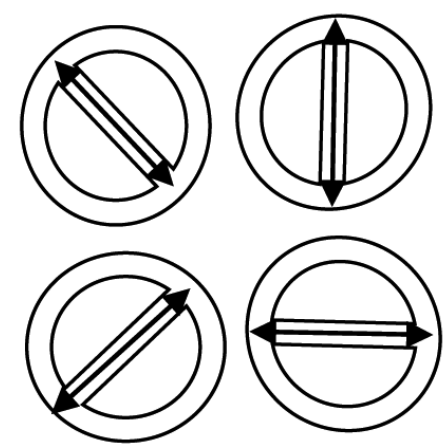

Puc. 3. Блок из четырёх элементов матрицы твист-ячеек с различной поляризацией луча на выходе благодаря различному углу твист-закрутки

Задавая различные комбинации поляризации и интенсивности света, падающего на дифракционную решётку из оптически анизотропного материала, можно добиться чрезвычайно широкого разнообразия возможных распределений интенсивности и поляризации в дифракционной картине.

\section{Выводы}

Разработана конструкция и методика использования ячеек ЖК с электроуправляемыми оптическими свойствами, регулирующих поляризацию луча све- 
та, падающего на дифракционную решётку, актуальных для элементной базы средств отображения и обработки информации.

Разработана конструкция и методика использования дифракционной решётки.

Работа выполнена при поддержке проекта РФФИ и Правительства Московской области № 17-47-500752.

\section{ЛИТЕРАТУРА}

1. Беляев В.В., Мащенко В.И., Соломатин А.С., Чаусов Д.Н. Заявка на патент РФ № 2015107901 от 10.03.2015 // Способ получения смеси жидкого кристалла с полимером для дисплейной техники и оптоэлектроники.

2. Жаркова Г.М., Сонин А.С. Жидкокристаллические композиты. Новосибирск: Наука. 1994. $214 \mathrm{c}$.

3. Мащенко В.И., Алексеев А.Н., Картавенко Т.В., Оленин А.В. Патент РФ №2473216 // Способ получения масс для лепки с биоцидными свойствами.

4. Соломатин А.С. Влияние профиля микрорельефа периодических анизотропных структур на их дифракционные свойства // Вестник Московского государственного областного университета. Серия: Физика-Математика. 2016. № 1. C. $74-87$.

5. Соломатин А.С. Линзы на основе жидких кристаллов с неоднородным радиальным распределением директора // Вестник Московского государственного областного университета. Серия: Физика-Математика. 2016. № 3. С. 37-45.

6. Соломатин А.С., Беляев В.В. Ориентационные и оптические свойства сферических доменов жидкого кристалла с центральной ориентирующей и внешней неориентирующей поверхностью // Вестник Московского государственного областного университета. Серия: Физика-Математика. 2016. № 4. С. 32-42.

7. Bedjaouia L., Gogibusb N., Ewenb B., Pakulab T., Coqueretc X., Benmounaa M., Maschkec U. Preferential solvation of the eutectic mixture of liquid crystals E7 in a polysiloxane // Polymer. 2004. Vol. 45. P. 6555-6560.

8. Lekkerkerker H.W., Verhoefl A.A. Droplet snap-off in fluids with nematic liquid crystalline ordering. // New Journal of Physics. 2012. Vol. 14. P. 12-20.

9. Parab S.S., Malik M.K., Deshmukh R.R. Dielectric relaxation and electro-optical switching behavior of nematic liquid crystal dispersed in poly(methyl methacrylate) // Journal of NonCrystalline Solids. 2012. Vol. 358. No. 18-19. P. 2713-2722.

10. Ralesh K., Kikuchi H., Stark M., Guckenberger R., Kajiyama T. Morphology and Interface of Liquid Crystal / Polymer Composite System at Nano Scale: A Model Investigation // Mol. Cryst. Liq. Cryst. 1999. Vol. 329. P. 171-180.

11. Su Y.-C., Chu C.-C., Chang W.-T., Hsiao V.K.S. Characterization of optically switchable holographic polymer-dispersed liquid crystal transmission gratings // Optical Materials. 2011. Vol. 34. No. 1. P. 251-255.

\section{REFERENCES}

1. Belyaev V.V., Mashchenko V.I., Solomatin AS, Chausov D.N. [Patent application of the Russian Federation no. 2015107901 of 10.03.2015] In: Sposob polucheniya smesi zhidkogo kristalla s polimerom dlya displeinoi tekhniki i optoelektroniki [Method of obtaining a mixture of a liquid crystal with a polymer for display technology and optoelectronics]. 
2. Zharkova G.M., Sonin A.S. Zhidkokristallicheskie kompozity [Liquid crystal composites]. Novosibirsk, Nauka Publ.. 1994. 214 p.

3. Mashchenko V.I., Alekseev A.N., Kartavenko T.V., Olenin A.V. [Patent of the Russian Federation no. 2473216]. In: Sposob polucheniya mass dlya lepki s biotsidnymi svoystvami. [Method for obtaining masses for modeling with biocidal properties].

4. Solomatin A.S. [Influence of the microrelief profile of periodic anisotropic structures on their diffraction properties]. In: Vestnik Moskovskogo gosudarstvennogo oblastnogo universiteta. Seriya: Fizika-Matematika [Bulletin of the Moscow Region State University. Series: Physics and Mathematics], 2016, no. 1, pp. 74-87.

5. Solomatin A.S. [Lenses based on liquid crystals with a non-uniform radial distribution of the director]. In: Vestnik Moskovskogo gosudarstvennogo oblastnogo universiteta. Seriya: Fizika-Matematika [Bulletin of the Moscow Region State University. Series: Physics and Mathematics], 2016, no. 3, pp. 37-45.

6. Solomatin A.S., Belyaev V.V. [Orientational and optical properties of the domains of the liquid crystal with a central orienting and external non-orienting surface]. In: Vestnik Moskovskogo gosudarstvennogo oblastnogo universiteta. Seriya: Fizika-Matematika [Bulletin of the Moscow Region State University. Series: Physics and Mathematics], 2016, no. 4, pp. 32-42.

7. Bedjaouia L., Gogibusb N., Ewenb B., Pakulab T., Coqueretc X., Benmounaa M., Maschkec U. Preferential solvation of the eutectic mixture of liquid crystals E7 in a polysiloxane. In: Polymer, 2004, vol. 45, pp. 6555-6560.

8. Lekkerkerker H.W., Verhoefl A.A. Droplet snap-off in fluids with nematic liquid crystalline ordering. In: New Journal of Physics, 2012, vol. 14, pp. 12-20.

9. Parab S.S., Malik M.K., Deshmukh R.R. Dielectric relaxation and electro-optical switching behavior of nematic liquid crystal dispersed in poly(methyl methacrylate). In: Journal of Non-Crystalline Solids, 2012, vol. 358, no. 18-19, pp. 2713-2722.

10. Ralesh K., Kikuchi H., Stark M., Guckenberger R., Kajiyama T. Morphology and Interface of Liquid Crystal. Polymer Composite System at Nano Scale: A Model Investigation. In: Mol. Cryst. Liq. Cryst., 1999, vol. 329, pp. 171-180.

11. Su Y.-C., Chu C.-C., Chang W.-T., Hsiao V.K.S. Characterization of optically switchable holographic polymer-dispersed liquid crystal transmission gratings. In: Optical Materials, 2011, vol. 34, no. 1, pp. 251-255.

\section{ИНФОРМАЦИЯ ОБ АВТОРАХ}

Соломатин Алексей Сергеевич - кандидат физико-математических наук, инженер учебно-научной лаборатории теоретической и прикладной нанотехнологии Московского государственного областного университета;

e-mail: Sotrudnica_UNC@mail.ru

Мащенко Владимир Игоревич - кандидат химических наук, старший научный сотрудник учебно-научной лаборатории теоретической и прикладной нанотехнологии Московского государственного областного университета;

e-mail:mashchenko@genebee.msu.su

Беляев Виктор Васильевич - доктор технических наук, профессор, заведующий кафедрой теоретической физики Московского государственного областного университета; e-mail:vic_belyaev@mail.ru 
Маргарян Акоп Левонович - кандидат физико-математических наук, старший научный сотрудник центра полупроводниковых приборов и нанотехнологий Ереванского государственного университета, Республика Армения;

e-mail: marhakob@ysu.am

Акопян Нуне Грантовна - младший научный сотрудник центра полупроводниковых приборов и нанотехнологий Ереванского государственного университета, Республика Армения;

e-mail: nune.hakobyan@ysu.am

\section{INFORMATION ABOUT THE AUTHORS}

Aleksei S. Solomatin - PhD in Physics and Mathematics, engineer of the Education \& Science Lab for Theoretical and Applied Nanotechnology, Moscow Region State University; e-mail: Sotrudnica_UNC@mail.ru

Vladimir I. Mashchenko - PhD in Chemistry, senior researcher of the Education \& Science Lab for Theoretical and Applied Nanotechnology, Moscow Region State University; e-mail: mashchenko@genebee.msu.su

Victor V. Belyaev - Doctor in Engineering, professor, head of the Department of Theoretical Physics, Moscow Region State University;

e-mail: vic_belyaev@mail.ru

Akob L. Margaryan - PhD in Physics and Mathematics, senior researcher of the Center of Semiconductor Devices and Nanotechnologies, Yerevan State University, Republic of Armenia; e-mail:marhakob@ysu.am

Nune H. Hakobyan - junior researcher of the Center of Semiconductor Devices and Nanotechnologies, Yerevan State University, Republic of Armenia; e-mail: nune.hakobyan@ysu.am

\section{ПРАВИЛЬНАЯ ССЫЛКА НА СТАТЬЮ}

Соломатин А.С, Мащенко В.И., Беляев В.В., Маргарян А.Л., Акопян Н.Г. Управляемые дифракционные элементы на основе жидкокристаллических композитов // Вестник Московского государственного областного университета. Серия: Физика-Математика. 2017. № 3. С. $76-83$.

DOI: $10.18384 / 2310-7251-2017-3-76-83$

\section{CORRECT REFERENCE TO THE ARTICLE}

Solomatin A.S., Mashchenko V.I., Belyaev V.V., Margaryan A.L., Hakobyan N.H. Controllable LC-Composite Diffractive Optical Elements. In: Bulletin of Moscow Region State University. Series: Physics and Mathematics, 2017, no. 3, pp. 76-83.

DOI: 10.18384/2310-7251-2017-3-76-83 